\title{
Describing the Chinese HIV Surveillance System and the Influences of Political Structures and Social Stigma
}

\author{
Lei Zhang ${ }^{*}, 1$ Eric Pui Fung Chow ${ }^{1}$, Jun Zhang ${ }^{2}$, Jun Jing ${ }^{2}$ and David P. Wilson ${ }^{1}$ \\ ${ }^{1}$ Kirby Institute, University of New South Wales, Sydney, Australia \\ ${ }^{2}$ Research Center for Public Health, Tsinghua University, Beijing, China
}

\begin{abstract}
China's public health surveillance system for HIV was established in late 1980s and has evolved significantly during the past three decades. With the gradually changing mode of HIV transmission from sharing of intravenous injecting equipment to sexual exposure and the rapid spread of HIV infection among Chinese homosexual men in recent years, an efficient and comprehensive population-level surveillance system for describing epidemics trends and risk behaviours associated with HIV acquisition are essential for effective public health interventions for HIV. The current review describes the overall strength of the Chinese HIV surveillance system and its structural weaknesses from a political and social perspective. The HIV surveillance system in China has undergone substantial revamping leading to a comprehensive, timely and efficient reporting system. However, large data gaps and lack of quality control and sharing of information obstruct the full performance of the system. This is largely due to fragmented authoritarianism brought about by the underlying political structure. Social stigma and discrimination in health institutes are also key barriers for further improvements of HIV diagnosis and surveillance in China.
\end{abstract}

Keywords: HIV surveillance, political structure, fragmented authoritarianism, social stigma, China.

\section{BACKGROUND}

By the end of 2009 it was estimated that 740,000 (0.560.92 million) people in China were living with HIV [1]. This estimate was obtained by using the Workbook Method recommended by UNAIDS based on the numbers of newly diagnosed HIV cases and applying related mortality rates reported by the current HIV sentinel surveillance system. The overall national HIV prevalence is estimated to be $0.057 \%(0.042-0.071 \%)$ among the Chinese population [2, 3 ]. However, data from the national sentinel surveillance for HIV/AIDS indicated magnitudes and trends in HIV prevalence vary substantially across different at-risk populations: e.g. $0.6 \%$ HIV prevalence among female sex workers (FSW) in 2009 [2] and increases from 1.4\% in 2001 to $5.3 \%$ in 2009 among men who have sex with men (MSM) $[4,5]$, and from $5.9 \%$ in 2002 to $9.3 \%$ in 2009 among injecting drug users (IDU) [2,6]. The profile of HIV epidemics in China have also been gradually changing in mode of transmission. In the early 1990s, the main driver of HIV transmission was needle sharing among IDU [6-9]; however, sexual transmission has now become the primary mode of transmission in recent years [2, 10-12]. The proportion of newly diagnosed HIV cases due to sexual transmission increased from $49.8 \%$ in 2005 to $74.7 \%$ in 2009 and newly diagnosed cases attributed to homosexual contact has substantially increased from $12.2 \%$ in 2007 to $32.5 \%$ in $2009[1,10]$.

Effective public health interventions for HIV rely on accurate and timely information on the extent and patterns of

\footnotetext{
*Address correspondence to this author at the CFI Building, Corner of West and Boundary Streets, Darlinghurst, Sydney NSW 2010, Australia; Tel: +61 29385 0900; Fax: +61 29385 0920; E-mail: lzhang@nchecr.unsw.edu.au
}

spread of infection. As such, an efficient and comprehensive population-level surveillance system for describing epidemics trends and risk behaviours associated with HIV acquisition are essential [13]. Previous studies have provided reviews of the current HIV surveillance system in China, mainly from a functional and epidemiological perspective [14-16]. In contrast, the current review aims to describe the overall strength of the Chinese HIV surveillance system and its structural weaknesses from a political and social perspective.

\section{Development of HIV Surveillance Systems in China}

Prior to 2004, newly diagnosed HIV/AIDS cases were reported through the National Disease Reporting System (NDRS) (see Table 1 for a list of reportable items in China). The system consists of multiple level of reporting. Villages, being the lowest level of administrative, collected HIV epidemiological data in their allocated areas and reported to prevention units in township hospitals. From the prevention units, HIV data were further sent through county health and epidemic-prevention stations (EPS) to provincial centers and forwarded to the Chinese Academy of Preventive Medicine. With the support from the Ministry of Health and cooperation of provincial centers of health and epidemic prevention, a computerized system had worked effectively to network the monitoring of HIV epidemics at various levels in China and information transfer experienced no substantial changes for about 25 years. However, all communicable disease surveillance systems in China underwent significant upgrade in 2004.

The severe acute respiratory syndrome (SARS) outbreak in 2003-2004 exposed the great deficiencies in both timeliness and completeness of infectious disease reporting 
Table 1. Items Reportable on a Diagnosed HIV Case Report Form

\begin{tabular}{|c|}
\hline Demographic Information \\
\hline - Name \\
\hline - Date of birth \\
\hline - Ethnic group \\
\hline - Marital status \\
\hline - Education level \\
\hline Disease Information \\
\hline - HIV positivity \\
\hline - $\quad$ Clinical diagnosis of AIDS $^{\dagger}$ \\
\hline Clinical HIV Diagnosis Test \\
\hline - Source of the blood sample ${ }^{*}$ \\
\hline - Status of the confirmation test \\
\hline - Confirmation test date and location \\
\hline History of High-Risk Behaviors, (Ever Involved in or Had) \\
\hline - Intravenous drug usage \\
\hline - Extramarital sexual activities \\
\hline - HIV-positive regular sexual partner \\
\hline - Sexual acts with MSM \\
\hline - Blood donation \\
\hline - Receiving blood transfer \\
\hline - Positive maternal HIV status \\
\hline - Occupational exposure to HIV \\
\hline - Major surgery \\
\hline Likely Route of Transmission \\
\hline - $\quad$ Sharing of injection equipment \\
\hline - Heterosexual transmission \\
\hline - Homosexual transmission \\
\hline - Blood contacts (donation/reception) \\
\hline - Mother-to-child transmission \\
\hline - Occupational exposure \\
\hline Reporting Organization \\
\hline - Name of the organisation \\
\hline - Name of the reporting clinician \\
\hline - Contact details and report date \\
\hline $\begin{array}{l}\text { Without specifying disease stages. } \\
\text { Circumstances which the blood sample was drawn, including pre-surgery testing, pre- } \\
\text { narital testing, voluntary blood donation, paid blood donation, tests for children with } \\
\text { ositive maternal status, detention center personnel testing, pregnancy blood testing, } \\
\text { ccupational exposure testing, sexual health clinic testing, voluntary counseling and } \\
\text { esting, new army recruits testing, migration body check, testing for discordant couples, } \\
\text { scientific surveys, testing for entertainment personnel and others. }\end{array}$ \\
\hline
\end{tabular}

in NDRS [17]. This substantially changed the landscape of infectious diseases surveillance in China. Just 6 months after the SARS epidemic subsided, the China Center for Disease Control (China CDC) launched the China Information
System for Disease Control and Prevention (CISDCP) [18]. The system is an information platform constructed mainly with an infectious diseases orientation, aiming to improve timeliness of case reports, completeness and accuracy of surveillance data, ability for early detection of outbreaks and to provide direct information sharing among various levels of CDCs and health authorities, replacing the previous NDRS. The upgrade of overall infectious disease surveillance systems in China directly led to the construction of web-based HIV/AIDS case reporting system. Under this reporting system, grassroot hospitals remain the primary source of HIV epidemiological information. All diagnosed and confirmed HIV-infected cases admitted to hospitals are obliged to report through the web-based reporting system to China CDC.

Improving from the previous step-wise hierarchical reporting framework, the new web-based reporting system provided the ability for direct reporting of diagnosed HIV cases from the grassroot hospitals to the centralised database in Chinese $\mathrm{MOH}$ through CISDCP (Fig. 1). That is, once disease cases are entered into the system, all levels of CDC can acquire the data 'live' according to their assigned access privileges [19]. This substantially reduced the average reporting period from 7-8 days to less than 2 days. In addition to their assumed responsibility for authenticating the HIV disease information in their administrative regions, municipal and provincial CDCs are required to report to their corresponding Department of Health (DOHs) and Bureau of Health (BOHs) and to form networks with local research bodies, universities and other health organisations. At the top of the hierarchy, the National Center for HIV/AIDS Prevention and Control (NCAIDS) of China CDC is the overseeing organisation for assembling and analysing all HIV disease information and then presenting a final report to China $\mathrm{MOH}$. China $\mathrm{MOH}$ remains the only legitimate office to disseminate HIV/AIDS-related information to the public, but provincial and municipal CDCs are also authorised to release information under the supervision and approval of China $\mathrm{MOH}[20,21]$. China $\mathrm{MOH}$ enacts health policies, designs prevention guidelines and intervention strategies based on the surveillance reports provided by NCAIDS, once a consensus agreement is established between $\mathrm{MOH}$ and the Chinese government, feedback recommendations and policies are issued to all lower levels of $\mathrm{MOH}$ for execution. Since the feedback information does not go through the webbased information pool, hospitals at grassroots level may have to wait for a considerable period of time to receive recommendations and guidelines from China $\mathrm{MOH}$.

By the year 2009, 8563 laboratories (8273 screening laboratories, 254 confirmatory laboratories, 35 confirmatory central laboratories and 1 National AIDS Reference Laboratory) and 84 laboratories hospitals are capable of carrying out CD4 and HIV viral load testing in China [22]. Confirmed diagnosed HIV cases were reported back to referring clinical sites then further integrated into the central database through CISDCP. The hospital-based surveillance in China has cumulatively diagnosed 326,000 people living AIDS and a total of 65,481 people are currently receiving ART in 2009 [23], which accounted for $62.4 \%$ of the AIDS cases [2]. 

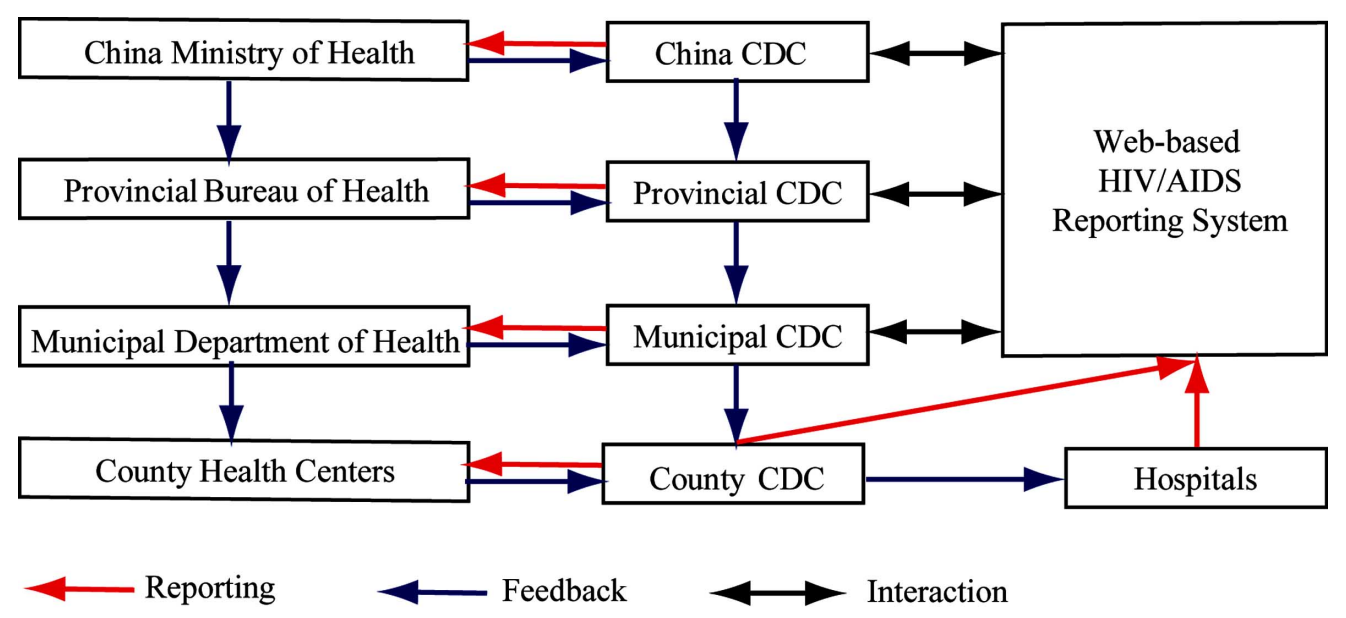

Fig. (1). Schematic diagram of flow of information for HIV surveillance in China.

\section{Sentinel Surveillance of HIV in China}

The hospital-based surveillance relies on PLHIV to attend hospital to be diagnosed and cases subsequently reported. Complementing this passive reporting, active surveillance for HIV was adopted in 1995 and further expanded in 2003 to include second generation behavioral surveillance in China according to WHO recommendations $[24,25]$. While the existing hospital-based diagnosis system provides HIV diagnosis and treatment data, sentinel surveillance collects information on HIV prevalence, incidence and the risk behaviours of at-risk populations. In China, HIV sentinel surveillance is collaboratively supervised by the Chinese MOH and WHO. In 2000, surveillance pilot sites targeting IDU, FSW, male clients and the general female population were established in Fujian, Xinjiang, Guangxi, Shanxi, Yunnan and Sichuan. The approach utilised 21 surveillance points in these provinces and from every surveillance point 400 people are sampled twice each year to estimate the prevalence, incidence rate and risk behaviours of the targeted population [26]. The sampling and reporting for each round is completed within 4 weeks. In 2002, China issued "Standards for HIV Surveillance and HIV/AIDS" and "STD Comprehensive Surveillance Guideline" to provide guidelines to standardise the practice of HIV surveillance. In 2009, the number of sentinel sites in China increased to 1318 , covering 14 at-risk population groups $[2,14,27]$. Since the establishment of the web-based HIV reporting system in 2004, sentinel surveillance data is integrated with disease information reported from hospitals. This approach established a comprehensive database for data collection, analysis and sharing and enables high-level comparison and integration of epidemiological and behavioural surveillance data [14]. This further allows the conduct of evaluation and forecasting activities for guiding the formulation of public health intervention strategies.

HIV sentinel surveillance has detected the changing trends and patterns of HIV epidemics in China, from transmission related to injecting practices to sexual transmission, especially homosexual transmission. Further, surveillance data have revealed that the proportion of IDU among all drug users has increased from $42 \%$ in 1994 [28, 29] to $53 \%$ in $2001[29,30]$ and $69 \%$ in 2009 [31]. In addition, during 1997-2009, the proportion of IDU who report sharing needles increased from $25 \%$ to $38 \%$ [32]. Among FSW, consistent condom use in commercial sexual contacts in the last month increased from $10 \%$ in 1995 to $62 \%$ in 2009 , and the proportion who reported never using condoms decreased from $70.6 \%$ to $2.0 \%$ during the same period $[14,33]$.

\section{Fragmented Authoritarianism and HIV Surveillance in China}

The current HIV surveillance system in China appears to have improved in its timeliness and effectiveness for providing useful HIV disease information and regular behavioural monitoring of at-risk populations in China. However, under-utilisation and lack of transparency of information outside certain authorised channels are possibly the greatest hindrances to optimal use of the current system. Since accessibility to health data in China is largely limited to a small group of authorised personnel at the top of the administrative hierarchy, it often requires extensive bargaining and bureaucratic procedures to obtain epidemiological and behavioural indicators that are commonly accessibly in other countries. Hence, large amount of surveillance data remains in CISDCP and cannot be utilised widely for the purpose of HIV public health research, broader program evaluation and planning of control strategies. A transparent HIV surveillance system across all stakeholders, including policy makers, health officials, community health organisation leaders and HIV researchers, is beneficial for the entire HIV sector in the evaluation and implementation of HIV prevention and intervention strategies [34]. To understand barriers to optimal utilisation of available systems it is essential to first understand the political structure in China and its influences on surveillance. Although HIV surveillance is considered as a purely public health issue in many countries, issues related to HIV in China are highly sensitive and politically motivated $[13,35$, 36].

Authoritarianism describes any political structure in which overall authority is concentrated in the hands of a single leader or a small group of elite. Prior to economic reform in China, the ultimate political powers were mastered by the chairman of state, who is simultaneously the president of the party and chief commander of the army. Political 
decisions and national policies were implemented strictly "from top to bottom" through a pyramidal administrative structure (Fig. 2a). During the process of political decentralisation initiated by the economic reform in China, provincial governments and ministerial institutions were given high degrees of autonomy to stimulate economic growth. Although the central government remains at the peak of the power hierarchy, opportunities for competition and bargaining between governments and institutions became possible. This phenomenon was coined "fragmented authoritarianism" by Lampton in 1987 [37]. As a result of fragmented authoritarianism, government authority below the very peak of the Beijing central government is fragmented and disjointed [37, 38]. As the highest administrative body, the central government does not generally dictate the execution of policies by provincial governments. Between equal level governments or governmental institutions, and even between local and central governments, there exists a large space for competition and cooperation, which leads to extensive bargaining concerning policy execution and project development of common interests (Fig. 2b). Fragmented authoritarianism in China can be viewed as the mixed product of traditional authoritarianism and demands for increase in political freedom triggered by the economic reform.

The fragmented authoritarian political structure directly affects the HIV surveillance system in China. First, fragmented authoritarianism leads to the absence of accountability in vertical administration of CDCs and lack of cooperation among CDCs on the same level. In this structure, the central China $\mathrm{MOH}$ cannot exercise direct supervision over the provincial MOHs, which retain the authority of personnel appointment, organisation structure and budget distribution. Conversely, policy immobility in inferior governments can only be overcome by the intervention of a superior government with the authority to

(a) Authoritarianism

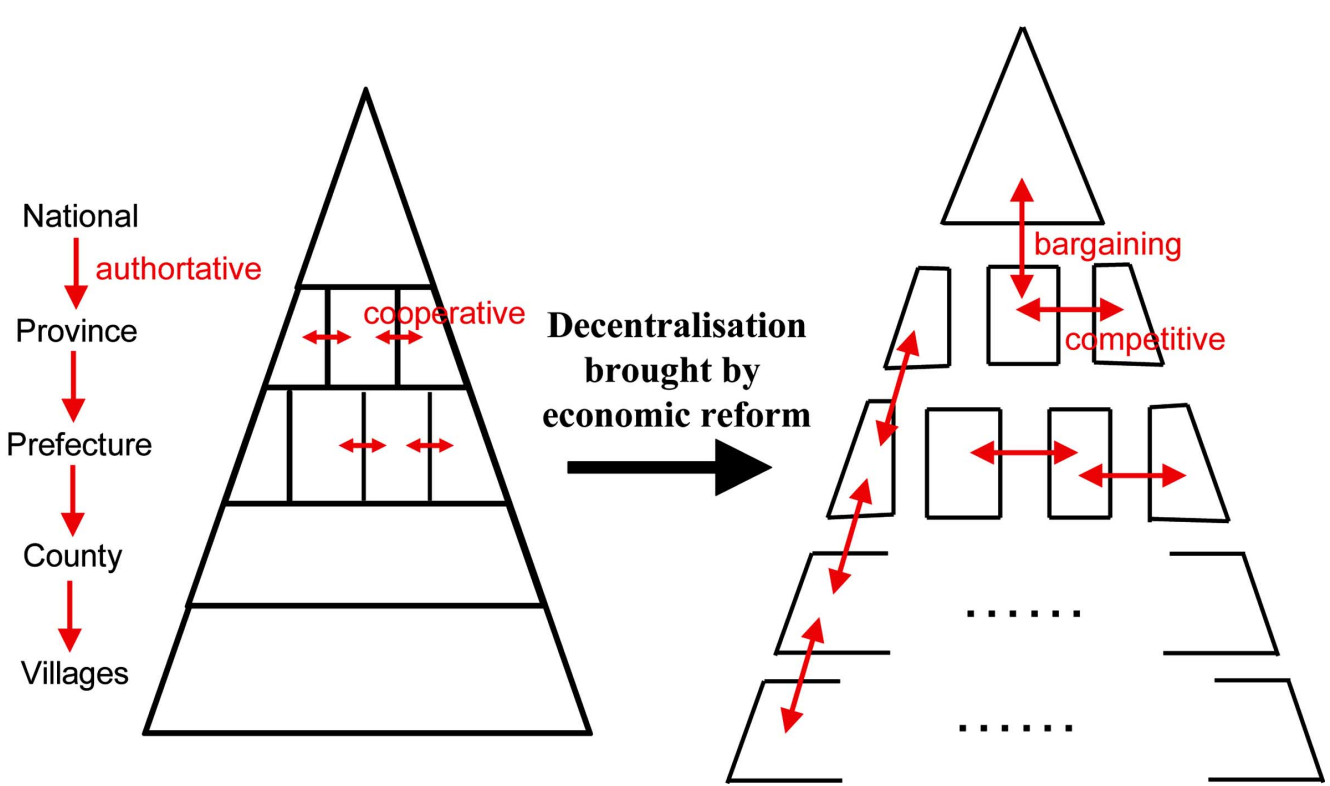

resolve conflicting interests. In other words, lower-level governments tend to shift their policy overload to the upper levels in order to avoid assuming accountabilities [38]. Consequently, a large number of responsibilities and agenda items accumulate at the upper end of the political hierarchy. Therefore, China CDC, as a subordinate organisation of China $\mathrm{MOH}$, is caught in an uneasy position whereby it is unable to exercise its designated authority and simultaneously overloaded with responsibilities which it is not designated to bear. This is reflected by the large amount of collected but unanalysed HIV data accumulated in NCAIDS in central China CDC. As pointed out by Sun, et al., the amount of HIV surveillance data from diagnoses, treatment, laboratory tests and behavioural surveillance overflows the organizational capacity of NCAIDS [14]. Apart from key indicators that require to be distributed to $\mathrm{MOH}$ and the general public, most of the surveillance data is under-utilised [14]. Although the web-based HIV reporting system provides an advanced technology base for data reporting and collection, the dissemination of related summary and policy to direct HIV prevention and care strategies remain slow and inefficient. In addition, such a fragmented system makes it difficult to authenticate the quality of the data, as intermediate levels of CDCs do not assume accountability for the accuracy of reported data. An evaluation of HIV surveillance systems by Loo et al., indicated that although the Chinese HIV surveillance system obtained high scores for flexibility and timeliness across in its surveillance activities, representativeness and completeness of data are poor in its case reporting [34]. Discrepancies are found between the HIV laboratory testing algorithm stated in protocols and the actual procedures implemented, which results in gaps in the data and reductions in quality [39].

Second, a fragmented authoritarian political structure inevitably leads to a fragmented information system with little openness and systematic mechanisms for synthesis. As

\section{(b) Fragmented Authoritarianism}

Fig. (2). Change of political relationship between institutions at the same and different administrative levels in China prior to, and post, economic reform. 
previously analysed, lower-level CDCs can only access HIV information within its own jurisdiction, which represents only a subset of the information pool of the higher-level CDCs. As there is little information sharing, even between neighbour CDCs on the same level, these information subsets become isolated information islands which can only be integrated by their superiors. In this way, the central China CDC at the top of the administration pyramid has the ultimate authority of data collection, assembling, analysis and distribution. Hence, information openness is strongly and solely dependent on the decision of central China CDC and there exists little monitoring mechanisms to ensure the accuracy of both the reported and published information. In the current political structure, all levels of government are only accountable to their upper-level administrations, but not to the general public. The absence of genuine engagement of civil society groups, including the media and the scientific communities, and the expectations and pressures from higher-level authorities, often results in a results-oriented policy implementation, such that local officials tend to manipulate nonscientific and arbitrary results to satisfy their superiors perfunctorily [40]. In this view, ensuring the accuracy of reported disease information should be a high priority in the future development of HIV surveillance in China.

\section{Social Stigma and HIV Surveillance in China}

Social stigma against PLHIV is common in China. Misconceptions about HIV transmission and prejudice towards PLHIV are widespread among the general population. HIV-positive individuals are often denied employment, education and necessary medical services [41] and are thus more likely not to disclose their HIV status [42, 43]. A recent study by Zhou in 2008 shows that the healthcare expenses for HIV-positive individuals were greatly elevated by social discrimination, and their available social resources were substantially reduced in the presence of social stigma [44]. The study further indicated that PLHIV often perceive healthcare institutions as an indispensable source of social support and one of the few places where they may be willing to disclose their HIV-positive status [44]. However, a separate survey among health workers in hospitals demonstrated that HIV stigmatisation remains as the major health-seeking barriers for PLHIV in China [45]. According to a UNAIDS study in $2009,15 \%$ of surveyed participants indicated that their HIV status was disclosed by health-care professionals to others without their consent [46]. According to the latest available statistics, there have been 326,000 cumulative HIV/AIDS cases in China, including 54,000 deaths, by the end of 2009 [23]. This accounts for only $44 \%$ of the estimated number of PLHIV at that time. The percentage of undiagnosed cases cannot be improved upon if the barrier of stigma against PLHIV from health institutions is not removed. In addition, PLHIV that belongs to at-risk groups, especially MSM, are subjected to greater social stigma due their identity and sexual orientation. Recent study indicated that only $14-45 \%$ of Chinese MSM underwent HIV testing in the past 12 months during 20072009 [2, 47-53]. Also, it is estimated that only $12-15 \%$ of HIV-positive MSM are diagnosed based on an estimate among MSM in Yunnan province in 2009 [54]. Due to the hidden nature of MSM, sentinel surveillance targeting MSM was also required to substantial scale-up. Although the number of sentinel surveillance sites targeting MSM has increased from 4 in 2006 to 25 sites in 2009 [1, 14], both epidemic and behavioural surveillance for MSM remains insufficient and limited in many parts of China. Reducing social stigma and discrimination is necessary in improving HIV surveillance in China.

\section{CONCLUSION}

In summary, the HIV surveillance system in China has undergone substantial revamping that leading to a comprehensive, timely and efficient reporting system. However, large data gaps and lack of quality control and sharing of information obstruct the full performance of the system. This is largely due to fragmented authoritarianism brought about by the underlying political structure. Social stigma and discrimination in health institutes are also key barriers for further improvements of HIV diagnosis and surveillance in China. The insufficient biological and behavioural surveillance for Chinese MSM may result in underestimation of the actual prevalence and disease burden among this population.

\section{ACKNOWLEDGEMENT}

The study was funded by Vice Chancellor Fellowship, the University of New South Wales, 2010-2012.

\section{CONFLICT OF INTEREST}

The authors confirm that this article content has no conflicts of interest.

\section{REFERENCES}

[1] Wang N, Wang L, Wu ZY, et al. Estimating the number of people living with HIV/AIDS in China: 2003-09. Int J Epidemiol 2010; 39: ii21-ii28.

[2] Ministry of Health of the People's Republic of China. China 2010 UNGASS Country Progress Report Beijing, China: Ministry of Health 2010.

[3] Sheng L, Cao WK. HIV/AIDS epidemiology and prevention in China. Chin Med J (Engl) 2008; 121(13): 1230-6.

[4] Chow EPF, Wilson DP, Zhang J, Jing J, Zhang L. HIV prevalence is increasing among men who have sex with men in China: findings from a review and meta-analysis. Sex Transm Dis 2011; 38: 84557.

[5] Tang WM, Ding JP, Yan HJ, Huan XP, Yang HT, Zhao JK. Sexual behaviors and HIV/syphilis infection among men who have sex with men: a meta analysis of data collected between 2001 and 2006 in the Chinese mainland. Chin J AIDS STD 2008; 14(5): 471-4, 88.

[6] Qian HZ, Vermund SH, Wang N. Risk of HIV/AIDS in China: subpopulations of special importance. Sex Transm Infect 2005; 81(6): 442-7.

[7] Office of the State Council Working Committee on AIDS China Progress on implementing UNGASS declaration of commitment in China 2005.

[8] Cohen DA, Wu SY, Farley TA. Comparing the cost-effectiveness of HIV prevention interventions. J Acquir Immune Defic Syndr 2004; 37(3): 1404-14.

[9] Qian HZ, Schumacher JE, Chen HT, Ruan YH. Injection drug use and HIV/AIDS in China: review of current situation, prevention and policy implications. Harm Reduct J 2006; 3: 4.

[10] Ministry of Health of the People's Republic of China. UNGASS Country Progress Report P. R. China (2006-2007): Beijing, China: Ministry of Health 2008.

[11] Watts J. Sex, drugs, and HIV/AIDS in China. Lancet 2008; 371(9607): 103-4.

[12] Lu L, Jia M, Ma Y, et al. The changing face of HIV in China. Nature 2008; 455(7213): 609-11. 
[13] Wu Z, Wang Y, Detels R, Rotheram-Borus MJ. China AIDS policy implementation: reversing the HIV/AIDS epidemic by 2015. Int J Epidemiol 2010; 39(Suppl 2): ii1-3.

[14] Sun X, Wang N, Li D, et al. The development of HIV/AIDS surveillance in China. AIDS 2007; 21(Suppl 8): S33-8.

[15] Wang L, Wang N. HIV/AIDS epidemic and the development of comprehensive surveillance system in China with challenges. Chin Med J (Engl) 2010; 123(23): 3495-500.

[16] Wu ZY. Current challenge of AIDS epidemic surveillance in China. Dis Surveill 2009; 24(11): 819-21.

[17] Lam WK, Zhong NS, Tan WC. Overview on SARS in Asia and the world. Respirology 2003; 8(Suppl): S2-5.

[18] Sha L, Li Y, Zhu Y, Ling XD, HongMei L, Wang Q. A sampling survey on syringe exchange and methadone maintenance treatment among drug abusers in Yunnan province. Chinese J AIDS/STD 2008; 14(3): 238-9.

[19] Jin S, Jiang T, Ma J. Brief Introduction of Chinese Infectious Disease Detection and Reporting Information System. China Digital Med 2006; 1(1): 20-2.

[20] China CDC. Information Release Guidelines for Infectious Diseases Epidemic and Public Health Contigencies. 2006. Available from: http://www.chinacdc.net.cn/n2724 42/n272530/n2 75462/n275477/n292891/14228.html [Cited: 200814 Nov].

[21] Li Z. Encyclopedia on the PRC State Secrets Law. ChangChun: Jilin People's Publishers 1999.

[22] Jiang Y, Qiu M, Zhang G, et al. Quality assurance in the HIV/AIDS laboratory network of China. Int J Epidemiol 2010; 39(Suppl 2): ii72-ii78

[23] Ministry of Health of the People's Republic of China, Joint United Nations Programme on HIV/AIDS, World Health Organization. 2009 Estimates for the HIV/AIDS Epidemic in China. Beijing, China 2010

[24] UNAIDS WHO. Guidelines for second generation HIV Surveillance for HIV: the next decade (2000). Geneva: World Health Organization and Joint United Nations Programme on HIV/AIDS 2000.

[25] Kretzschmar M, Zhang W, Mikolajczyk RT, et al. Regional differences in HIV prevalence among drug users in China: potential for future spread of HIV? BMC Infect Dis 2008; 8: 108.

[26] The UN Theme Group on HIV/AIDS in China. HIV/AIDS: China's Titanic Peril - 2001 Update of the AIDS Situation and Needs Assessment Report 2002.

[27] Wang L, Wang L, Ding ZW, et al. HIV prevalence among populations at risk, using sentinel surveillance data from 1995 to 2009 in China. Chin J Epidemiol 2011; 32(1): 20-6.

[28] Yang H, Li X, Stanton B, et al. Heterosexual transmission of HIV in China: a systematic review of behavioral studies in the past two decades. Sex Transm Dis 2005; 32(5): 270-80.

[29] China CDC. National HIV/AIDS Sentinel Surveillance Report in 2003. Beijing: China CDC \& National Sentinel Surveillance Group 2004.

[30] Chu TX, Levy JA. Injection drug use and HIV/AIDS transmission in China. Cell Res 2005; 15(11-12): 865-9.

[31] Bao YP, Liu ZM. Systematic review of HIV and HCV infection among drug users in China. Int J STD AIDS 2009; 20(6): 399-405.

[32] Guo W, Qu SQ, Ding ZW, Yan RX, Li DM, Wang L. Situations and trends of HIV and syphilis infections among drug users in China, 1995-2009. Zhonghua Liu Xing Bing Xue Za Zhi 2010; 31(6): 666-9.

[33] Fu SG, Sun LN, Meng XJ, Ding ZW, Wang L. Surveillance of AIDS among female sex workers in China 1995-2009. Dis Surveill 2010; 25(11): 850-3.
[34] Loo V, Kim E, Monterroso E, et al. Improving HIV surveillance systems: Country experiences and a proposal for evaluation framework. J HIV/AIDS Surveill Epidemiol 2010; 2(1): 10-8.

[35] Liu Y, Wu Z, Mao Y, Rou K, Wang L, Zhang F. Quantitatively monitoring AIDS policy implementation in China. Int J Epidemiol. 2010; 39(Suppl 2): ii90-6.

[36] He XP, Rofel L. "I Am AIDS": Living with HIV/AIDS in China. Positions: East Asia Cultures Critique 2010; 18(2): 511-36.

[37] Lampton DM. Policy Implementation in Post-Mao China. Berkeley and Los Angeles, CA: University of California Press 1987.

[38] Huang Y. The SARS Epidemic and its Aftermath in China: A Political Perspective. In: Knobler S, Ed. Learning from SARS: Preparing for the Next Disease Outbreak. Washington: The National Academies Press 2004. pp. 116-36.

[39] Shah N, Kim A, Liu J, Lu W, Peng L, Zhao J, Eds. Evaluation of the Guangdong HIV/AIDS surveillance system, China. In 2nd Global HIV/AIDS Surveillance Meet; 2009; Bangkok, Thailand.

[40] Zhang L. Building a Better Infectious Disease Surveillance System for China: An evaluation from a political perspective. Berlin: VDM Verlag 2009.

[41] Burki TK. Discrimination against people with HIV persists in China. Lancet 2011; 377(9762): 286-7.

[42] Yoshioka MR, Schustack A. Disclosure of HIV status: cultural issues of Asian patients. AIDS Patient Care STDS 2001; 15(2): 7782.

[43] Li L, Sun S, Wu Z, Wu S, Lin C, Yan Z. Disclosure of HIV status is a family matter: field notes from China. J Fam Psychol 2007; 21(2): 307-14.

[44] Zhou YR. Help-seeking in a context of AIDS stigma: understanding the healthcare needs of people with HIV/AIDS in China. Health Soc Care Community 2009; 17(2): 202-8.

[45] Lieber E, Li L, Wu Z, Rotheram-Borus MJ, Guan J. HIV/STD stigmatization fears as health-seeking barriers in China. AIDS Behav 2006; 10: 463-71.

[46] UNAIDS. The China Stigma Index Report. Beijing: UNAIDS2009.

[47] Zeng G, Xiao Y, Xu P, Feng N, Jin CR, Lu F. Evaluation of effect of community-based HIV/AIDS interventions among men who have sex with men in eighteen cities, China. Zhonghua Yu Fang Yi Xue Za Zhi 2009; 43(11): 977-80.

[48] Wei C, Ruan S, Zhao J, Yang H, Zhu Y, Raymond HF. Which Chinese men who have sex with men miss out on HIV testing? Sex Transm Infect 2011; 87(3): 225-8.

[49] Sun M, Li D, Jin W, Jiang J, Guan L. Investigation on the Infection of HIV, HCV, Syphilis and HBV among MSM in Dalian City in 2008. Prev Med Tribune 2009; 15(11): 1074 - 5.

[50] Zhong F, Lin P, Xu H, et al. Possible increase in HIV and syphilis prevalence among men who have sex with men in Guangzhou, China: results from a respondent-driven sampling survey. AIDS Behav 2011; 15: 1058-66.

[51] Weng YQ, Bai Y. Surveillance on the high risk behaviors among 239 men who have sex with men. J Appl Prev Med 2009; 15(3): 152-3.

[52] Xiao Y, Ding X, Li C, Liu J, Sun J, Jia Y. Prevalence and correlates of HIV and syphilis infections among men who have sex with men in Chongqing Municipality, China. Sex Transm Dis 2009; 36(10): 647-56.

[53] Yang SJ, Mu HT, Li XQ, et al. Survey of the knowledge, attitude, and practice on aids among MSM population in Urumchi. Mod Prev Med 2007; 34(20): 4624-5.

[54] Chow EP, Wilson DP, Zhang L. The next era of HIV in China: rapidly spreading epidemics among men who have sex with men. J Acquir Immune Defic Syndr 2010; 55(4): e32-3; author reply e3-4. 